\title{
الحيّات السامة وعضات الحيات في منطقة جازان - المملكة العربية السعودية
}

\author{
سالم محفوظ بسيس 1، 2 ، عبدالعزيز حسن المالكي 1 و ابراهيم حسين غزواني 1 \\ 1 1 قسم الأحياء ، كلية العلوم، جامعة جازان، المملكة العربية السعودية

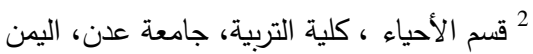 \\ البريد الالكتروني: s.busais@gmail.com
}



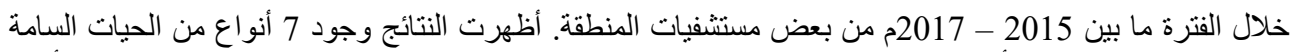



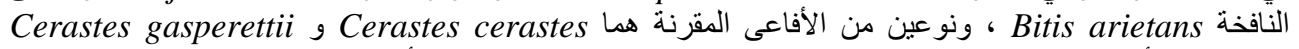

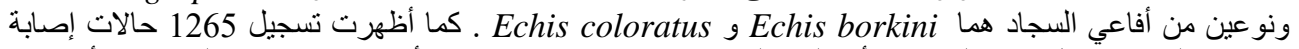

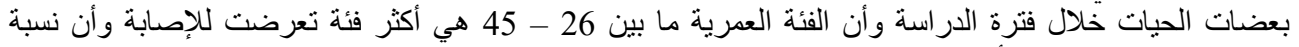
الإصابة عند الذكور كانت أعلى مما عليه عند الإناث بنسبة 3.2 : 1 ـ

كلمات مفتاحية: الأفاعي، الثعابين، عضات الحيات، جازان، المملكة العربية السعودية.
\end{abstract}

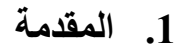

الحيات السامة التي لها رأس مميز عن الجسم بعنق واضح، ولها ذيل يتميز بشكل ملفت عن الجسم [1]. توجد الحيات في كل البيئات عدا في القطبين. وتضم ما يزيد عن 3800 نوع سام و غير سام [2]. و وعلى الرغم من أن الأفاعي والثعابين السامة تشكل ما نسبته أقل من 30\% من مجموع الحيات في العالم [3]، إلا أن المشاكل الصحية و الاقتصادية التي تسببها هذه الأنواع السامة كبيرة. وتمتاز المملكة العربية السعودية بوجود 51 نوع من الحيات 9 أنواع منها هي من الحيّات
الحيّات من طائفة الزواحف وتتنمي الى رنبة الحرشفيات التي تضم تحت رتبتي السحالي والحيّات. والحيّات اسم عام يشمل كل الأفاعي و الثعابين، مفردها حَيّة Snake ويُطلق هذا الاسم على أي نوع من الحيّات، سواء أَكانت أفاعي، أو ثعابين، سامة، أو غير سامة. أما الثعبان فهو يطلق على الحيات الممتدة الطويلة التي لا، Serpent يتميز رأسها عن عنقها بوضوح، وإن وجد لها أنياب فهي ثابتة غير متحركة، أما الأفعى Viper فهو يطلق على 


$$
\text { سالم محفوظ بسيس ، عبدالعزيز حسن المالكي و ابراهيم حسين غزواني }
$$

أجريت في بعض مناطق المملكة العربية السعودية كالرياض [13 ؛16 ؛17؛ 18]، و عسير [19]، و الباحة [20]، وحائل [21]. من الدراسات السابقة في المملكة العربية السعودية نلاحظ أن مناطق عديدة من المملكة لم يُجرَ عليها در اسة عن حالات الإصابة بعضات الحيّات. ونظرًا لأهمية هذا الموضوع، وقلة الدراسات في هذا الجانب في الكثير من مناطق المملكة وأحيانا عدم توفرها؛ كان الهدف من هذا البحث معرفة عدد حالات التعرض لعضات الحيّات في منطقة جازان، وتحديد عدد الأنواع السامة من الحيات في المنطقة، كي تكون خطوة أولى في تسليط الضوء على هذا الموضوع المهم في حياة الناس في منطقة جاز ان بدرجة أساسية و المملكة بشكل عام.

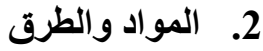

تمت هذه الدراسة على مرحلتين الأولى: تصنيف أنواع الحيات السامة في منطقة جازان. والمرحلة الثانية جمع الإحصائيات عن حالات الإصابة بعضات الحيات من خلال زيارة أهم مستشفيات المنطقة وجمع المعلومات منها في الفترة ما بين 2015 - 2017.

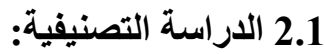

تم جمع الحيّات من مناطق مختلفة من منطقة جازان، بالإضافة إلى عينات كانت محفوظة مُسبقًا في معمل قسم الأحياء في جامعة جاز ان، بالإضافة إلى ما ذكِر في المر اجع

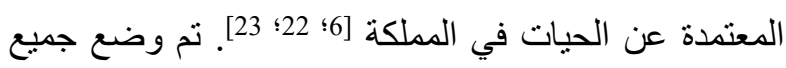
العينات في أوعية زجاجية وبلاستيكية وحقن الحيّات و غمر ها بمادة حافظة هي ايثانول 70\%، أو فورمالين 4\% وتم حفظها في قسم الأحياء في جامعة جازان، وقد تم تصنيف الحيّات اعتمادًا على جملة من الصفات الظاهرية موضحة في المر اجع المعتبرة [6؛ 22].
السامة البرية [6:4]. لهذا قامت المملكة العربية السعودية

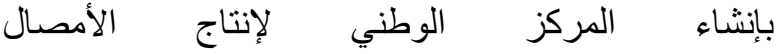
واللقاحات: Antivenom and Vaccine (NAVPC) Production Center” أمصال ثنائي الفاعلية يستخدم ضد سموم الكوبرا العربية ، Walterinesia aegyptia والصل الأسود Naja haje وأمصال متعددة الفاعلية يستخدم ضد سموم ستة أنواع من الأفاعي والثعابين المسجلة في المملكة وهي: الأفعى النافثة


وأفعى السجاد الشرقي Echis coloratus، وأفعى الطفي المنشارية Echis carinatus، ونوعين من الصلال هما الكوبرا العربية Naja haje، و الكوبرا المصرية أو الصل الأسود Walterinesia aegyptia [5]. وتنتشر أنواع مختلفة من الحيّات بنسب متفاوتة في مناطق المملكة، إلا أن منطقة جازان تتميز بوجود أكبر عدد من الأنواع فيها [6]. تقع منطقة جازان في الجنوب الغربي من المملكة العربية

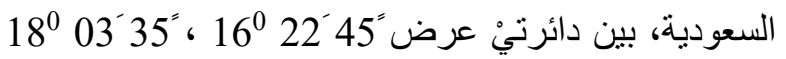

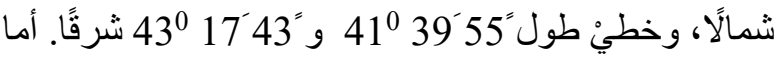
مساحتها فتصل الى 12435 كم2 [7]. وتعتبر الإصابة بعضات الحيات "Snakebites" من التحديات الكبيرة التي تو اجه الدول المختلفة في العالم، حيث يصل معدل الاصـابة بعضاتها من 2.1 - 5.5 مليون حالة سنويًّا، يتوفى منها ما لها يقارب لئ 125000 شخص [8؛ 10؛ 11]، وتحتل الهند المرتبة الأولى في معدل الإصابة بعضات الحيات حيث تصل إلى أكثر من 200000 حالة سنويَّا، قد يتعرض ربع هذه الحالات الى الوفاة [12]، بينما تقل في الولايات المتحدة فتصل الإصابة السنوية تقريبًا إلى 8000 حالة [13]. إن الييانات عن حالات الإصابة بعضات الحيّات في الدول العربية غير دقيقة لقلة الإحصائيات عنها، وقد أجريت بعض الدراسات في دول مثل الأردن و المغرب [1، 14، 15]، كما 
Bitis منطقة جازان خمسة أنواع هي: الأفعى النافخة 2.2 (الدراسة الميدانية:

Cerastes arietans ونوعين من الأفاعى المقرنة هما Cerastes gasperettii cerastes السجاد هما Echis borkini و Echis coloratus. أظهرت النتائج الإحصائية لعدد حالات الاصابة بعضات الحيات في مستشفيات جاز ان المشمولة خلال فترة الدراسة ما مجمو عه 1265 حالة بمعدل 121.67 حالة سنويًّا (جدول 1)، و أن أعلى تسجيل في المناطق الساحلية كان في مستشفى أبو عريش العام بمعدل 160.7 حالات سنويا، أما المناطق الجبلية فكانت في مستشفى بني مالك بمعدل 108.7 حالات سنويا (الجدول 1). أما عن أعلى حالات الإصابة بين الجنسين فقد سجلت عند الذكور بنسبة 3.2 : 1 (شكل 1). أما في الفئات العمرية فقد وجد أن الفئة العمرية ما بين 26 45 هي أعلى فئة إصابة بعضات الحيات، و أقلها كانت عند الفئة العمرية الأقل من 15 سنة (جدول 1). أما عن حالات الاصابة بين المواطنين السعوديين و المقيمين فقد بلغت بنسبة أكبر عند المواطنين بمعدل 1.43 : 1 (شكل 2). أما عن حالات الوفيات التي تم احصاؤها خلال فترة الدراسة فقد بلغت حالة و احدة فقط .



شكل 1: يوضح عدد حالات الإصابة بعضات الحيات المسجلة بين الأكور والإناث خلال الفترة 2015 - 2017 م.
تمت زيارات ميدانية الى أهم مستشفيات المنطقة وهي: مستشفى الملك فهد المركزي، ومستشفى بني مالك العام، ومستشفى أبوعريش العام، ومستشفى صبيا العام. اعتمدت الدر اسة على الإحصائيات المسجلة في تلك المستشفيات في الفترة ما بين 2015 - 2017م، مسلطة الضوء على المعلومات التالية: جنس المصاب، العمر، المنطقة السكنية التي أصيب فيها، منطقة الإصابة في الجسد، ووقت الإصابة، والحالة قبل العلاج، والحالة بعد العلاج، ونوع العلاج المقدم للمصاب، والجنسية، و عدد الوفيات.

\section{3 - 3. - (النتائج}

أظهرت النتائج أن هنالك 7 أنواع من التعابين والأفاعي الخطيرة على حياة الإنسان، تعود إلى ثناث فصائل هي فصيلة الثعابين اللامعة Lamprophiidae ومنها النوع الذي يدعى بالأفعى الحافرة المعروفة باسم الأسود الخبيث Elapidae Atractaspis andersonii ممثلة بـالكوبرا العربية Naja arabica، وأخيرا فصيلة الأفاعي Viperidae، وتعتبر هذه الفصيلة من أكبر فصائل الحيات السامة من حيث عدد الأنواع حيث سجل منها في جدول 1: يظهر حالات الإصابة بعضات الحيات المسجلة في بعض مستثفيات منطقة جاز ان خلال الفترة 2015 - 2017 م.

\begin{tabular}{|c|c|c|c|c|c|c|c|}
\hline الوفيات & 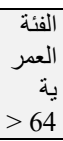 & 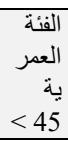 & $\begin{array}{r}\text { الفئة } \\
\text { العمر } \\
\text { الفئة } \\
\end{array}$ & 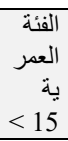 & العمرية & لي الإجما & السمتشفى \\
\hline 1 & 33 & 117 & 16 & 33 & 46 & 199 & المركزي فهذ \\
\hline 0 & - & - & - & - & - & 326 & بني مالك \\
\hline 0 & 81 & 232 & 128 & 41 & 0 & 482 & أبو العام \\
\hline 0 & - & - & - & - & - & 258 & صبيا العام \\
\hline
\end{tabular}

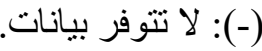




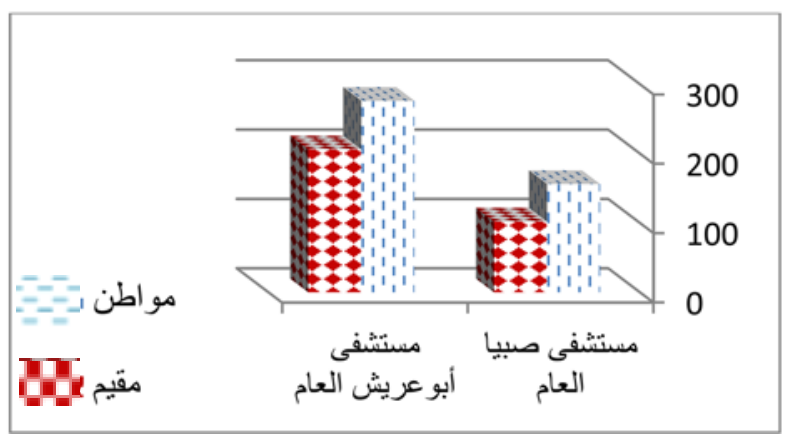

وحائل والجوف تم تسجيل أربع أنو اع فقط من الحيّات السامة الخطبرة [13 ؛ 26؛ 27]. ومن المُسلم به؛ أن تحديد أنو اع الحيّات السامة في المنطقة ضروريَّا جدًا لتوفير المصل الخاص بها، إذ أنّ لكل نوع من الحيات السامة لقاحًا خاصًا به لعلاج المصاب بعضة الحية يستخرج من نفس نوع الحية السامة، و إلا فإن حياة المصاب ستكون معرضة للخطر، خاصة إذا لم يحسن التعامل مع المصاب، أو قد نم استعمال مضاد سمّي غير مناسب لنوع الحية السامة، وذللك أن التركيب الكيميائي لكل سُمّ يختلف من نوع لآخر [1؛ 6؛ 22؛ 25]. من خلال الإحصائيات وجد ارتفاع في المعدل السنوي لحالات الإصابة بعضات الحيات في جازان مقارنة بغيرها من مناطق المملكة، وقد يعود السبب في ذلك إلى كثرة أنواع الحيات في المنطقة و إلى العادات المجتمعية لدى سكان جازان حيث تعتبر منطقة جاز ان منطقة زراعية، فالناس على احتكالك مباشر بالبيئة من خلال عملهم في الأنشطة الزراعية، أو من حيث الخروج الى البر للتنزه، مما يجعلهم أكثر عرضة للإصابة بعضات الحيات. و على الرغم من كثرة حالات الإصابة بعضات الأفاعي و الثعابين في جاز ان إلا أن حالات الوفيات منخفضة بشكل ملحوظ بلغت حوالي 0.21\% و هي نسبة قليلة جدَّا إذا ما قورنت بدر اسات سابقة تمت في بعض مناطق المملكة حيث سجلت في الرياض بنسبة 2.3\% [17]، وفي دراسة أخرى في الرياض كذلك بلغت النسبة 4.8\% [18]، ولكن نسبة الوفيات في دراسة
شكل 2: يوضح عدد حالات الإصابة بين المواطنين السعوديين و المقيمين خلال الفترة 2015 - 2017 م.

\section{المناقشة}

تشير الدراسات السابقة الى وجود 16 نوع من الثعابين و الأفاعي مسجلة في منطقة جاز ان [6؛ 22]، و هذا العدد كبير بالنسبة إلى منطقة صغيرة لا يتجاوز مساحتها 12435 كم²، ولكن نتيجة لتنوع التضاريس في منطقة جازان و التي تضم السهول و الجبال و الوديان و المناطق الزراعية و السبخات، فإن هذه البيئات المختلفة مع ما تحمله السيول من أفاعي وثعابين من خارج المنطقة، ومحدودية المناطق المأهولة بالسكان، كل هذه العوامل ساعدت على توفر الموطن المناسب لمعيشة وتنوع هذه الحيّات وانتشار ها، بالإضافة إلى قلة المفترسات و التي يأتي على رأسها الورل و الطبور الجارحة ـ التي عادةً ما تتعرض للإيذاء أو القتل من الناس و هي مفترسات عادةً تنشط نهارًا [24]، في حين أن نشاط أغلب الحيّات يكون ليلًا [25]، وبالمقابل يأتي كثرة الأنواع وتوفر الفرائس كالقوارض والزواحف الصغيرة والعلاجيم المنتشرة في المنطقة نتيجة للتنوع التضاريسي الكبير، و أحيانًا نتيجة للنشاط الإنساني المنمثل برمي القمامة في غير الأماكن المخصص لها؛ ساعد على وجود وفرة في الحيّات، وبالتالي نلاحظ كثرة أنواع الثعابين والأفاعي السامة في منطقة جازان عنها في أبي منطقة أخرى في المملكة العربية السعودية، بينما نجد على سبيل المثال أن في منطقة الرياض 
الأجزاء العليا أو السفلى في الجسم، ونوع العلاج المقدم، ونوع الأفعى، ونوع التسمم الناتج منها، والمنطقة التي جاء منها المصاب. لهذا نرى أنه لابد من تضمين هذه المعلومات في بيانات المستشفيات، وتوحيد البيانات في مستشفيات المملكة، وإثر الك خبراء في علم التصنيف في الدراسات الطبية المرتبطة بالحيوانات، حتى يتم تحديد النوع بشكل دقيق، وبالتالي يساعد ذلك في سرعة الثفاء وانخفاض تكاليف العلاج. ونوصي بإدراج التصنيف الجزيئي ضمن الأبحاث التصنيفية وتقديم الدعم لها حيث أنها مهمة جدًا في التمبيز بين الأنواع، و إيجاد علاقات التقارب بينها، خاصة تللك التي تتداخل فيها الصفات الثكلية، كما في حالة الأفاعي المقرنة من الجنس Cerastes، أو الجنس الآخر Atractaspis الخبيث، أو الجنس Walterinnesia المعروف باسم الصل الأسود أو الكوبرا المصرية، فمن خلال الدراسات الجزيئية سيتم الفصل في وضعيتها التصنيفية من حيث وجودها منمثلا بنوعين، أو بنوع واحد [24]. مع أن المركز الوطني لإنتاج الأمصال و اللقاحات ذكر أنواع الحيّات التي يستخلص سمّها كي تستخدم كأمصال، إلّا أنها لم تشمل كل الأنواع السامة Atractaspis المسجلة في المملكة مثل الأسود الخبيث andersonii Atractaspis engaddensis المملكة، كما لم يشمل الصّّل الأسود من نوع Walterinnesia morgani Echis من المملكة إن صحت نسبة النوع له، أو النوع الموجود في جنوب غرب المملكة والذي pyramidum صار اسمه E. burkini و هي أنواع سامة جدًا، فينبغي على المركز الوطني NAVPC تعديل الأسماء العلمية وتحديثها و إضافة ما ليس في قائمته، وتعديل اسم الكوبرا العربية من Naja arabica إلى الاسم العلمي الجديد. Naja haje
حديثة في الرياض انخفضت إلى 0.3\% [13]، أما في الباحة فقد بلغت 1.2\% [20]، وفي منطقة حائل فقد بلغت نسبة الوفيات 2.9\% [21]. وقد يعود السبب في انخفاض معدل الوفيات في جازان إلى زيادة و عي سكان المنطقة في التعامل مع حالات الإصابة بعضات الحيات، وكذلك الدور الفعال التي تقوم به وزارة الصحة بتقديمها خدمات ورعاية طبية متقدمة بالإضافة إلى الخدمات المقدمة من المركز الوطني لإنتاج الأمصال واللقاحات NAVPC الذي يعتمد على أفاعي وثعابين محلية لتوفير الأمصال المضادة للسموم من مما يساهم في التعجيل من شفاء المصابين. كما أظهرت النتائج أن نسبة إصابة الذكور أعلى من الإناث وهذا يتو افق مع النتائج السابقة التي أجريت في المملكة [13؛ 17؛ 18؛ 20] كما ينو افق كذلك مع نتائج أجريت في دول أخرى مثل المغرب والإمارات العربية المتحدة وسلطنة عمان و إيران و غير هم [11؛ 14؛ 15؛ 28]، وهذا يعود إلى أن الذكور في الغالب أكثر احتكاكًا بالطبيعة من الإناث، وكذللك هم أكبر جرأة في التعامل معها، وبالتالي يكونوا أكثر عرضة للإصابة بعضاتها. كما أظهرت الدراسة أن الفئة العمرية ما لـان بين 26 - 45 سنة هي أكبر فئة معرضة للإصـابة بعضات الحيات، وهي نتيجة تبدو طبيعية كون الأشخاص في هذا العمر هم في الغالب أكثر تعاملًا مع البيئة الطبيعية، وبالتالي فإن احتمالية مصادفتهم للأفاعي والثعابين والتعرض لهاب تكون عالية، وهذه نتيجة قد نوافقت كذللك مع الدراسات السابقة في المغرب و إيران [11؛ 14].

إن البيانات الإحصائية المسجلة في المستشفيات في منطقة جاز ان ليست متطابقة حيث تترك بعض التفاصيل في بعض المستشفيات، أو قد ينقصها بعض المعلومات التي لو توفرت ستسهر في تقديم صورة أوضح لحالات الإصابة بعضات الحيات، ومن البيانات المطلوبة موضع الإصابة هل هي في 
[1] عمرو، زهير ، راتب العوران ، نثأت حميدان. "الحيو انات السامة في الأردن، الحيات". الجران، الجمعية الدلكية لحماية الطبيعة. 127 صفحة (لأرن، (2012).

Uetz, P. "The Reptile Database". http://www.reptile-database.org, accessed July 3, 2020.

Hider, R.C., Karlsson, E., Namiraman, S. [3] "Separation and purification of toxins from snake venoms". In: Harvey, A.L. (Ed.), Snake Toxins. Pergamon Press, New York, pp. 1-34 (1991).

Al-Sadoon M. K. "Survey of the reptilian fauna of the Kingdom of Saudi Arabia. I. The snake fauna of the Central Region". $J$. Coll. Sci., King Saud University, 1 (1-2), 53 .- 59 (1989).

National Antivenom and Vaccine Production [5] Center. http://www.antivenom.center.com/ar/. accessed October 28, 2018 Gasperetti, J. "Snakes of Arabia". Fauna of [6] Saudi Arabia. 9: 169-450 (1988).

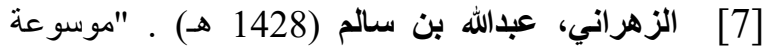
المملكة العربية السعودية، المجلد الحادي عشر، المالم منطقة

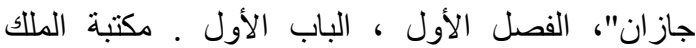
عبدالعزيز العامة (1988).

Kasturiratne A, Wickremasinghe A. R, de [8] Silva N, Gunawardena N. K, Pathmeswaran A, Premaratna R. "The global burden of snakebite: a literature analysis and modelling based on regional estimates of envenoming and deaths". PLoS .Med. 5 (11): 218 (2008).

Adukauskiene D, Varanauskiene E,

Adukauskaite A. "Venomous snakebites". Medicina (Kaunas). 47(8):461-7 (2011).

Rita P, Animesh D. K, Aninda M, Benoy [10]

G. K, Halder S. "Snake bite, snake venom, anti-venom and herbal antidote". A review. Int $J$ Res Ayurveda pharm. 2(4):1060-7 (2011).

Dehghani, R., D. Rabani, M.P. Shahi, M. [11] Jazayeri and M.S. Bidgoli. "Incidence of
ومن حسن توجهات المركز الوطني NAVPC أنه يعتمد في إنتاج الأمصال على حيوانات جُمعت من البيئة المحلية، وبالتالي فإن نسبة الدقة في العلاج تكون أعلى وفرصة

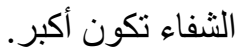

إن تحديد النوع بدقة ومتابعة الاسم العلمي الحديث لله و عمل الأمصال من هذه الأنواع المصنفة مطلب مهم جدًا لضمان صحة العلاج وسرعة الثفاء وخفض تكاليفه. ومن هنا يظهر أهمية متابعة الأسماء العلمية، والوقوف الى تحديثاتها تجنبًّا لأي خلطٍ قد يؤدي إلى انتاج مضـادات سمية غير فعّالة في علاج المصابين. ولعله من الأفضل إضافة اسم المنطقة للثعبان أو الأفعى الذي تم انتاج المصل منه ضمن بيانات المصل، خاصة إذا وضعنا في الحسبان أن التركيب الكيميائي للسم قد يتغير باختلاف المنطقة التي تم احضار الأفعى أو الثعبان منها لاختلاف نوعية الغذاء، وهنا يصبح وضع اسم المنطقة على اللقاح ضروري. كذللك نوصي بأن يوضع في قسم الطوارئ في المستشفيات ملصقات تعريفية بأنو اع الكائنات الستّامة في المنطقة، وعرضها على المصاب أو مر افقيه حتى يسهل من عملية التشخيص و العلاج. الشكر والتقدير

نتقدم بالثكر لجامعة جازان على تمويل هذا البحث ضمن مشروع علماء المستقبل 5 برقم (FS5-045)، و الذي حصل على المركز الثالث في الترتيب. والثكر موصول لرئيس قسم الأحياء، جامعة جاز ان، د. حسين الناشري، و عميد كلية العلوم د. زرّاق الفيفي على تسهيل الصعاب، وتوفير المعدل المناسب. كما نتوجه بالثكر الجزيل لمدراء المستشفيات المشمولة ضمن الدراسة وموظفي قسم الإحصاء لتسهيلهم جمع البيانات المطلوبة في هذه الدراسة.

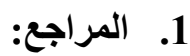


Al-Mohareb, F., Al-Sadoon, M. "Outcome [20] of snake bite in AlBaha". Ann. Saudi Med.

14, 26-29 (1994).

Mahaba, H.M. (2000). "Snake bites: [21] epidemiology, prevention, clinical presentation and management". Saudi Med. Journal. 20 (1), 66-68

Egan, D. "Snakes of Arabia". Motivate Publishing, Dubai, 208 pp (2007).

Sindaco R., Venchi A., Grieco C. "The [23] Reptiles of the Western Palearctic, Volume 2: Annotated Checklist and Distributional Atlas of the Snakes of Europe, North Africa, Middle East and Central Asia, with an Update to Volume ,1". Edizioni Belvedere, Latina, Italy (2013).

[24] بسيس، سالم محفوظ. "دراسة وصفية مع المفتاح

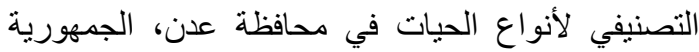
اليمنية". مجلة جامعة عدن للعلوم الطبيعية و التطبيقية، 18 النية التئية 567-557:(3)

[25] السعدون، محمد بن خالد (1425هـ). "أنواع العقارب (2014).

و الثعابين السامة في منطقة الجوف ـ دراسة تصنيفة التيفية

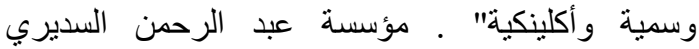

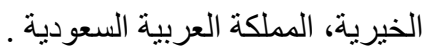

[26] السعدون ، محمد خالد ؛ سعود الفراج (1413هـ) .

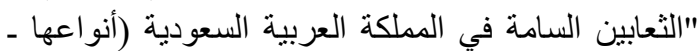

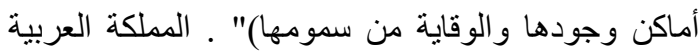

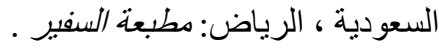

Al-Shammari, A. M. \& Busais, S.

"Distribution of Snakes in Ha'il Province, Saudi Arabia, with an Identification Key to the Species". Russian Journal of Herpetology. 27 (1), 5-10 (2020).

Alkaabi, J.M., Al Neyadi, M., Al Darei, [28] F., Al Mazrooei, M., Al Yazedi, J., Abdulle, A.M. "Terrestrial snake bites in the South East of the Arabian Peninsula: patient characteristics, clinical presentations, and management". PLOS ONE 6 (9), e24637 (2011).
Snake Bites in Kashan, Iran During an Eight Year Period (2004-2011)". Arch. Trauma Res., 1: 67-71 (2012).

Chippaux J. P. "Snake-bites: appraisal of [12] the global situation". Bull World Health Organ. 76 (5): 515-24 (1998).

Al-Sadoon, M. "Snake bite envenomation [13] in Riyadh province, of Saudi Arabia over the period (2005-2010)". Saudi Journal of

Biological Sciences. 22, 198-203 (2015).

Arfaoui, A., Hmimou, R., Ouammi, L., [14] Soulaymani, A., Mokhtari, A., Chafiq, F., Soulaymani-Bencheikh, R. "Epidemiological profile of snake bites in Morocco". J. Venom. Anim. Toxins Incl. Trop. Dis. 15 (4), 653-666 (2009).

Chafiq F., El Hattimy F., Rhalem N., [15] Chippaux JP., Soulaymani A., Mokhtari A., Soulaymani-Bencheikh R. "Snakebites notified to the poison control center of Morocco between 2009 and 2013". Journal of Venomous Animal and Toxins Including Tropical Diseases.22: 8 (2016).

Al-Sadoon, M. K., Abdo, N.M. "Fetal [16] envenomation by snake Atractaspis, newly recorded in the central region of Saudi Arabia". Journal of King Saud Univ. Sci. 3 (2), 123-131 (1990).

Al-Sadoon, M. K., Jarrar, B.A. "Study of [17] the frequency and incidence of scorpion stings and snake bites in Riyadh city". Journal of King Saudi Univ. Sci. 6 (2), 217-

266 (1994).

Al-Durihim, H., Al-Hussaini, M., Bin [18] Salih, S., Hassan, I., Harakati, M., Al Hajjaj, A. "Snake bite envenomation: experience at King Abdulaziz Medical City, Riyadh". EMHJ 16 (4):438-41 (2010). Malik, G.M. "Snake bites in adults from [19] the Asir region of southern Saudi Arabia". Am. J. Trop. Med. Hyg. 52, 314-317 (1995). 


\title{
Venomous Snakes and Snakebites in Jazan Region, KSA
}

\author{
Salem M. Busais ${ }^{1,2}$, Abdulaziz H. Al Maliki ${ }^{1}$ and Ibrahim H. Ghazwani ${ }^{1}$ \\ ${ }^{1}$ Department of Biology, Faculty of Science, Jazan University, P.O. Box: 1394, 45142 Jazan, KSA, \\ ${ }^{2}$ Department of Biology, Faculty of Education, Aden University, Yemen \\ E MAIL: s.busais@gmail.com
}

ABSTRACT: The poisonous snakes and snakebites in the Jazan Region were studied through the period of 20152017. The results revealed that there are seven species of poisonous snakes in the region of Jazan, namely: the Arabian Small-Scaled Burrowing Asp (Atractaspis andersonii), the Arabian cobra (Naja arabica), the Puff Adder (Bitis arietans), two species of Horned Vipers (Cerastes cerastes and Cerastes gasperettii), and two species of Carpet Viper (Echis borkini and Echis coloratus). In addition, 1265 cases were recorded during the study period. The people aged between 26 and 45 were the most affected age group. The percentage of infection in males was higher than females by 3.2: 1 .

Keywords: Serpents, Vipers, Snakebites, Jazan, Saudi Arabia. 
JKAU: Sci., Vol. 31.Number(2) pp: 51-58 (2019 A.D. / 1440 A.H.)

DOI: $10.4197 /$ Sci.31-2.5 